\title{
Sull'alternanza dei suffissi -iatra e -logo nella terminologia medica: pediatra e cardiologo
}

\author{
Manuela Cainelli
}

PUBBLICATO: 19 DICEMBRE 2008

\section{Quesito:}

Milena Bramardi si rivolge al nostro servizio di consulenza per avere chiarimenti sulla formazione delle parole nella terminologia medica; in particolare chiede perché il suffisso di alcuni specialisti è iatra (pediatra ecc.) e quello di altri -logo (cardiologo ecc.).

\section{Sull'alternanza dei suffissi -iatra e -logo nella terminologia medica: pediatra e cardiologo}

U na parte consistente del lessico italiano comprende i linguaggi settoriali, soprattutto d'ambito tecnico-specialistico, dotati di una propria terminologia specifica e impiegati nell'ambito di particolari attività. Per quanto riguarda la medicina, troviamo ad esempio termini quali cardiologo oppure pediatra. Il primo è composto da cardio- e-logo; il secondo invece da pedo- e -iatra. Il confisso cardio- o-cardio deriva dal greco kardia 'cuore' e costituisce il primo o secondo elemento di composti appartenenti al linguaggio medico (cardiologo appunto, oppure miocardio). Anche il confisso logo- o -logo può essere il primo o il secondo elemento di composti; esso è ricavato dal greco lógos 'parola, discorso' ed indica appunto 'linguaggio, parola' (in logopedia), oppure forma composti che designano persone che si occupano di una determinata disciplina (e dunque cardiologo). Primo elemento di composti appartenenti alla terminologia tecnico-specialistica è poi pedo-, ricavato, nel significato di 'fanciullo', dal greco pâis, paidós 'ragazzo'. Dal greco iatrós 'medico' deriva infine-iatra, quale secondo elemento di composti appartenenti alla terminologia medica (pediatra, psichiatra).

È pertanto possibile notare che è proprio della medicina far uso di composti di origine greca. In proposito, Maurizio Dardano osserva che "L'uso del greco nella formazione delle terminologie scientifiche è legato a vari fattori. Da un punto di vista strutturale il greco, più di altre lingue, ha una spiccata propensione per i meccanismi di prefissazione, suffissazione e soprattutto composizione [...]. Inoltre, come lingua di un popolo che è stato culla della civiltà occidentale, il greco è tradizionalmente legato a numerose scienze, tecniche e discipline. Nel greco esse hanno trovato il primo e fondamentale strumento di espressione; in greco si sono formati i primi linguaggi settoriali ad esse legati; alla lingua e cultura greca fanno tutt'ora riferimento concetti e categorie di quelle discipline che più guardano al loro passato, [...]" (M. Dardano, Formazione delle parole nelle terminologie tecnico-scientifiche. Introduzione, p. 579).

Il linguista Tullio De Mauro ci informa inoltre che la composizione costituisce un "procedimento di formazione di nuovi lessemi antico e generalmente vitale nelle lingue indoeuropee, dal sanscrito alle lingue germaniche, [...]. Il procedimento è stato largamente adottato dal latino scientifico internazionale e dai linguaggi tecnici e scientifici dell'Europa moderna" (T. De Mauro, La fabbrica delle parole. Il lessico e problemi di lessicologia, p. 152). Di qui, tale processo è dunque stato accolto nell'italiano, dando luogo alla creazione di elementi compositivi quali appunto i confissi, ossia "quegli elementi come logo- o -logo che nelle parole composte possono apparire sia al primo sia al secondo posto" (Ibid., p. I78). Si può dunque concludere, con Serianni, che "nel suo insieme la formazione delle 
parole risponde efficacemente alle esigenze comunicative del linguaggio medico. Spesso, sommando i significati delle varie componenti lessicali di un termine, è possibile risalire al significato dell'intera parola" (L. Serianni, Formazione delle parole nelle terminologie tecnico-scientifiche. Medicina, p. 586).

Tornando ai due casi proposti, il confisso -iatra è esclusivo del settore medico (psichiatra, odontoiatra), mentre -logo è condiviso da molti settori (dialettologo, antropologo). Come mai pediatra e non pedologo? In effetti c'è in italiano anche pedologia, variante di pediatria senza successo, anche perché insidiata da un diverso valore di pedo- dal greco pedon, che significa terreno, per cui il pedologo esiste ma è un geologo e non un medico. I dizionari attestano anche unlaringologo laddove ormai si usa otorinolaringoiatra. Perché sia prevalsa una forma invece di un'altra andrebbe ricostruito attraverso una documentazione sulla storia delle parole. Noi ci possiamo limitare a dire che erano entrambe possibili dal punto di vista della lingua.

Nota bibliografica:

- Maurizio Dardano, Formazione delle parole nelle terminologie tecnico-scientifiche. Introduzione, in La formazione delle parole in italiano, a cura di M. Grossmann e F. Rainer, Tübingen, Niemeyer, 2004.

- Tullio De Mauro, La fabbrica delle parole. Il lessico e problemi di lessicologia, Torino, UTET, 2005.

- Luca Serianni, Tecnicismi medici e farmacologici contemporanei, in Saggi di storia linguistica italiana, a cura di L. Serianni, Napoli, Morano, I989, pp. 38I-420.

- Luca Serianni, Italiani scritti, Bologna, il Mulino, 2003 (cap. 6, I linguaggi settoriali, pp. 79-88).

- Luca Serianni, Formazione delle parole nelle terminologie tecnico-scientifiche. Medicina, in La formazione delle parole in italiano, a cura di M. Grossmann e F. Rainer, Tübingen, Niemeyer, 2004.

\section{Cita come:}

Manuela Cainelli, Sull'alternanza dei suffissi-iatra e -logo nella terminologia medica: pediatra $e$ cardiologo , "Italiano digitale", 2008, XVI, 2021/1 (gennaio-marzo)

DOI: 10.35948/2532-9006/2021.6492

Copyright 2008 Accademia della Crusca

Pubblicato con licenza creative commons CC BY-NC-ND 